\title{
Comparison of weed spread models
}

\author{
R. I. Hickson ${ }^{1} \quad$ S. I. Barry ${ }^{2} \quad$ K. Stokes $^{3}$
}

(Received 30 July 2007; revised 18 December 2007)

\begin{abstract}
Numerous models of weed spread and growth exist in ecology. We compare four common models: reaction diffusion, integro-difference, 'scatter' model, and an 'occupation' model. We discuss their similarities, strengths and limitations. After verifying the equivalence of the integro-difference model, with a Gaussian kernel, against the reaction diffusion model, we show how to equate parameters of the different models. We also investigate the effect of the occupation model parameters on spread behaviour.
\end{abstract}

\section{Contents}

\section{Introduction}

See http://anziamj.austms.org.au/ojs/index.php/ANZIAMJ/article/view/339 for this article, (c) Austral. Mathematical Soc. 2008. Published January 9, 2008. ISSN 1446-8735 
2 Models

C327

2.1 Reaction diffusion . . . . . . . . . . . . . . . . . . C327

2.2 Integro-difference . . . . . . . . . . . . . . . . . . . C328

2.3 Scatter model . . . . . . . . . . . . . . . . . . . . . . C329

2.4 Occupation model . . . . . . . . . . . . . . . . C330

3 Simulation results

C331

4 Conclusion

C336

References

C337

\section{Introduction}

Weed spread is of financial concern as introduced plants invade agricultural land, decreasing productivity, and environmental concern as they modify the natural ecosystems in Australia [3]. Hence predicting weed spread facilitates proper management and control. Numerous models exist for the spatial growth and spread of weeds. Although there have been several reviews of weed spread $[1,4,7,8,9,10,11,15,16]$, to our knowledge there have been no direct numerical comparisons of reaction diffusion, integro-difference, 'scatter' and 'occupation' models. Here we discuss which parameters are equivalent for the reaction diffusion, integro-difference, and 'occupation' models, and highlight the differences between the 'scatter' and integro-difference models. We verify that the integro-difference equation, with a Gaussian distribution, is equivalent to the reaction diffusion model, as a test of the accuracy of our numerical implementations. We also investigate the effect of changing the occupation model parameters on the spread behaviour and discuss the similarities, strengths and limitations of the models.

Fisher [6] did some of the earliest work on the reaction diffusion model, including calculating the asymptotic wave speed. Skellam [18] was one of the 
first to use this Fisher model for an invasive ecological scenario. The general form of the reaction diffusion model [10] is

$$
\frac{\partial N}{\partial t}=\nabla \cdot(D \nabla N)+\gamma f(N),
$$

where $N(x, y, t)$ is the density function of plants at position $(x, y)$ at time $t$, $D(x, y, t)$ is the diffusivity, $\gamma(x, y, t)$ is the intrinsic growth rate and $f(N)$ is the growth rate function and takes many forms $[9,10,14,15,16]$, and usually $0 \leq f(N) \leq 1$.

The integro-difference equation was first used to model populations in genetics $[11,13,19,20]$ where Weinberger $[19,20]$ conducted a comprehensive wave speed analysis. This model is now commonly used to investigate plant spread rates $[9,11]$. The 'scatter' model uses the integro-difference equation with a stochastic finite dispersal kernel.

Various cellular automata simulations have been developed as a means of modelling plant spread, as summarised by Higgins and Richardson [8]. We concentrate on the work by Rees and Paynter [17] who developed an 'occupation' model for the spread of Scotch broom, which is an invasive shrub-like plant. The distinguishing feature of this work is the inclusion of a probability of disturbance.

In comparing these models we evaluate their consistency over three different criteria: the total population; the position of the travelling weed front down the centreline of the region as functions of time; and the speed of the weed front as a function of the growth term. Section 2 discusses the attributes, advantages and limitations of each model, emphasising important similarities. Section 3 matches the parameters of the models, using our simulations of typical weed spread scenarios to verify the equivalence of the integro-difference and reaction diffusion models and explore the effect of the probability of disturbance on the occupation model. 


\section{Models}

In all simulations we assume a homogeneous region, which may underestimate the speed of the weed front [7], with constant parameters, and one weed species with no stage structure; that is, the seed, seedling and age structure of the plant are not considered.

\section{$2.1 \quad$ Reaction diffusion}

The reaction diffusion model we use combines growth and diffusion $[1,8,10]$

$$
\frac{\partial N}{\partial t}=D\left(\frac{\partial^{2} N}{\partial x^{2}}+\frac{\partial^{2} N}{\partial y^{2}}\right)+\gamma f(N)
$$

where $N=N(x, y, t)$ is the density of plants [branches $/ \mathrm{m}^{2}$ ] at position $(x, y)[\mathrm{m}]$ and time $t$ [month], $D$ is the diffusivity $\left[\mathrm{m}^{2} /\right.$ month] and $\gamma$ is the intrinsic growth rate $\left[\right.$ month $\left.^{-1}\right]$. The units of 'branches' could easily be changed to 'individuals'. The growth rate function is chosen to include a common logistic expression [8]

$$
f(N)=N\left(1-\frac{N}{C}\right)
$$

where $C$ is the carrying capacity.

The diffusive term in equation (2) is equivalent to assuming a normally distributed (Gaussian) dispersal pattern [7, 8, 15]; however, experimental results indicate that dispersal patterns are usually leptokurtic $[8,14,16]$. That is, they have a long tail distribution, with plants spreading further than a Gaussian distribution; hence limiting the application of this model to plant spread. The asymptotic wave speed $c[7]$ in one spatial dimension, is

$$
c=\sqrt{4 \gamma D},
$$


and in two dimensions, the radial speed is

$$
c=\sqrt{4 \pi \gamma D} .
$$

\subsection{Integro-difference}

The integro-difference model is a combination of growth followed by dispersal in a discrete time cycle, where the dispersal pattern is governed by any kernel $[10,14,15]$. The model considers each position in space and determines what disperses to that position from every other position in the region. The integro-difference equation has the general form in one spatial dimension (with obvious extension to two dimensions) of

$$
N(x, t+\tau)=\int_{z} K(x, z) f(N(z, t)) d z,
$$

where $N(x, t)$ is the density of plants, $\tau$ is the discrete time step, $K(x, z)$ is the dispersal kernel from spatial point $z$ to $x$, and $f(N(z, t))$ has many forms $[10,14,15]$. We use equation (3) for consistency. Any dispersal kernel is possible, including combinations of kernels [15] which mimics the different spread mechanisms of plants. For example, seeds drop around the plant in a Gaussian distribution, but are spread leptokurtically by birds to distant locations. This model is equivalent to the reaction diffusion model when the dispersal kernel has a Gaussian distribution [15], which is depicted in Figure 1(a):

$$
K(x, z)=\frac{1}{\sqrt{4 \pi D \tau}} \exp \left(-\frac{(x-z)^{2}}{4 \pi D \tau}\right),
$$

where $D$ is directly equivalent to the diffusivity term of the reaction diffusion model, equation $(2)[2,12]$. The integro-difference model with non-Gaussian dispersal kernels has been shown [2] to be equivalent to fractional reaction diffusion equations, which are not discussed here. 

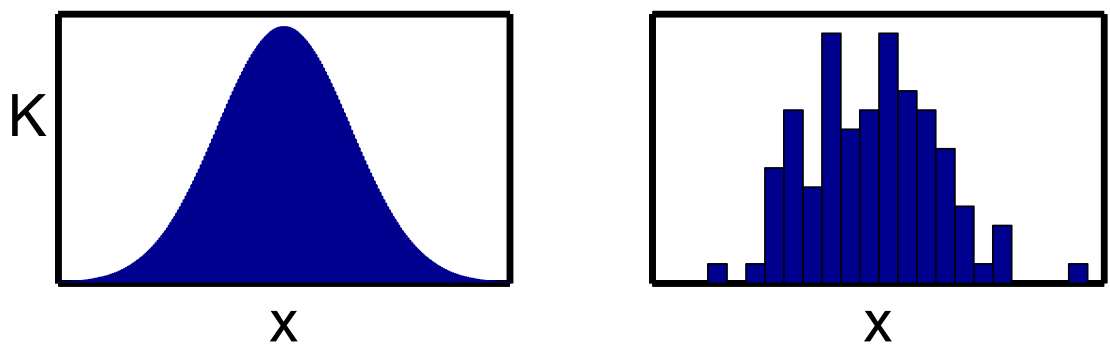

FiguRE 1: Gaussian dispersal kernels $K(x)$ for: (a) Integro-difference model; (b) Scatter model.

We make the common assumption that $K(x, z) \equiv K(x-z)$ implying that the region is spatially homogeneous, as the dispersal is only a function of the distance between two sites $[14,15]$. Kot et al. [9] show that when the dispersal kernel is given by equation (7), the wave speed for this model is given by equation (4). That is, with these assumptions the asymptotic wave speeds for the reaction diffusion and integro-difference models are identical. Asymptotic wave speeds for general growth rate functions, $f(N)$, have also been derived [9] but are not discussed here.

\subsection{Scatter model}

The scatter model we use is similar to the integro-difference model, equation (6), but it uses a rule based finite stochastic dispersal kernel as per cellular automata models. The difference between the kernels is depicted in Figure 1. That is, we assume plants arrive in finite groups as is common in ecology, for example birds will usually carry large quantities of seeds to approximately the same distant location. Normally, to model realistic spread, we would use a kernel with long distance dispersal events, however for this comparison we use a finite Gaussian distribution, shown in Figure 1(b), to better compare models. The result of the difference in the dispersal kernels 
is a more realistic, 'clumpy' spread pattern [12].

\subsection{Occupation model}

An occupation model is a cellular automata model. We concentrate on a particular example developed by Rees and Paynter [17] to model the spread of Scotch broom. A series of transition rules are used at each site to control the population density over time [8]. The model assumes a small enough spatial discretisation that a single plant occupies an entire site, hence making the plant density $N=0$ or 1 . The occupation model has numerous parameters: plants older than $A_{\text {min }}$ disperse $F$ seeds according to some kernel, assumed to be nearest neighbour, where $f_{h}$ is the probability a seed remains in the parent site. As per the model by Rees and Paynter [17], the lattice is rectangloid and the nearest neighbours are the surrounding eight cells. The decay of seeds over time is controlled by the probability $d$. The probability of a new plant is governed by two parameters: $g$, the probability a seed becomes a seedling; and $s$, the probability a seedling survives to the end of its first year. New plants can only grow if a site is disturbed, which occurs with probability $p_{\text {dist }}$. This mimics ecological cases where weeds cannot establish at sites already occupied by native species. When a plant reaches $A_{\max }$ years, it dies and the site is re-colonised with probability $p_{\text {so }}$.

The occupation model is easy to implement numerically and is appropriate for situations where "a single plant can influence invasion patterns" [8]. Since there is a single plant per site, it is best suited for small spatial scales [8], as large areas would be too computationally intensive. Modifying the assumption to include multiple plants per site would overcome this limitation. However, this would fundamentally change the model and is not examined here, but is the subject of further work. 


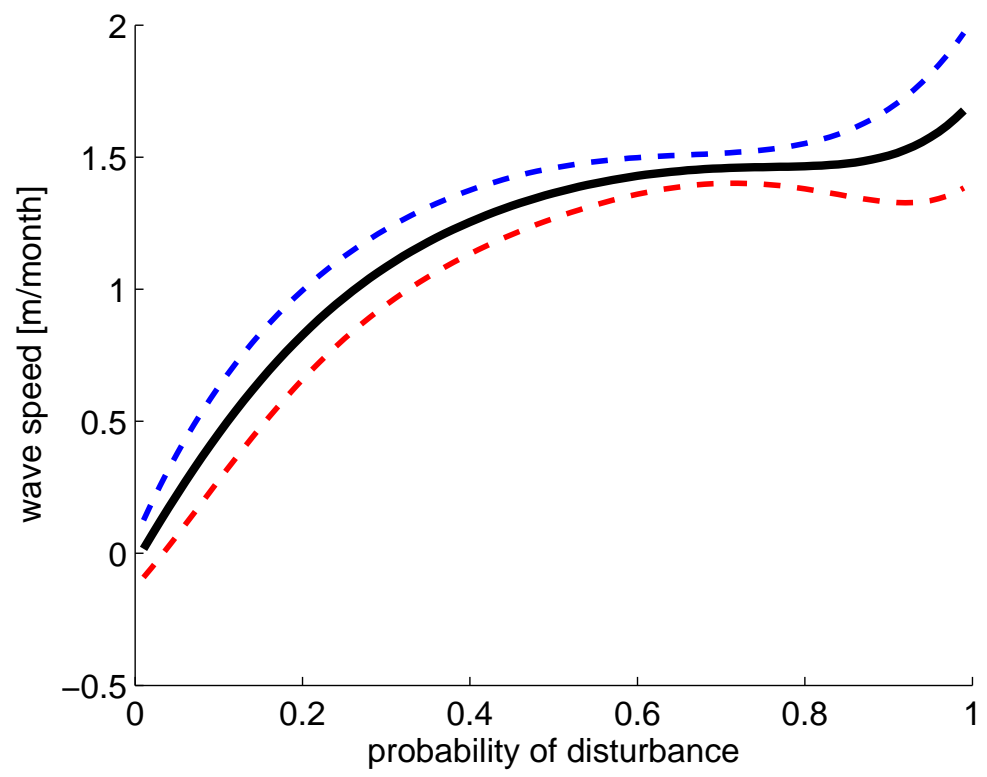

FIGURE 2: Wave speed of the occupation model as a function of the probability of disturbance, $p_{\text {dist }}$, with $95 \%$ confidence bounds (dashed lines).

\section{Simulation results}

Each of the models used a $112.5 \mathrm{~m} \times 112.5 \mathrm{~m}$ sized homogeneous region in the $(x, y)$ directions, discretised to a $75 \times 75$ grid. Initially a strip of plants with a density of 10 branches $/ \mathrm{m}^{2}$ were placed in the top $10 \%$ of the region. The side boundaries were wrapped to simulate an infinite region, so plants disappearing off the left of the region appear on the right and vice versa, as is common in the ecological literature [17]. The top boundary was insulated $(\partial N / \partial y=0)$ to prevent any loss of plants. No boundary condition was set at the bottom of the region as simulations were terminated before the weed front reached here. 
An important aspect of model comparison is matching the parameters. Therefore, since the reaction diffusion model is limited to a Gaussian dispersal kernel, the integro-difference and scatter models used only this distribution. In order to approximate the nearest neighbour distribution of the occupation model, the diffusivity, $D$, was set to the length of the grid in the $y$ direction squared divided by the size of the time step: $2.25 \mathrm{~m}^{2} /$ month. To match the growth term of the reaction diffusion and integro-difference models with the occupation model parameters we approximated the probabilities controlling the recruitment of a new plant so that $\gamma \approx g \times s$, as detailed in section 2.4.

The inclusion of the probability of disturbance in the occupation model provides a significant difference to the other models. For this reason we initially set $p_{\text {dist }}=0.8$ for Figures 3 to 5 , which means that a recruited plant is able to grow in most grids at every time step. For completeness, we calculated the speed of the weed front for the occupation model as a function of the probability of disturbance, as shown in Figure 2. The results depicted here were averaged over ten repetitions, with a standard deviation of $0.065 \mathrm{~m} /$ month.

For Figures 2, 3 and 4, the growth term $\gamma=0.1$. The occupation model values were (per year): $F=5600, d=0.5, g \times s=\gamma=0.1, f_{h}=0.6$, $A_{\min }=0, A_{\max } \approx \infty$, and $p_{\text {so }}=0.0$. Note the values chosen for the agedependent parameters result in an occupation model with no age structure. All stochastic model results were averaged over ten runs for all figures. The confidence bounds were omitted from these figures for clarity.

The standard deviations for Figure 3 were 433 plants for the scatter model, for the occupation model with $p_{\text {dist }}=0.8$ it was 246 plants, for $p_{\text {dist }}=0.2$ it was 1556 plants, and for $p_{\text {dist }}=0.1$ it was 858 plants. Figure 3 shows the total number of plants as a function of time, illustrating that the reaction diffusion, integro-difference and scatter models are mostly identical, which is to be expected since we specifically chose the parameters to match. The slight differences between the maximum values are due to a combination of discretisation errors in the integro-difference and scatter models, and the 


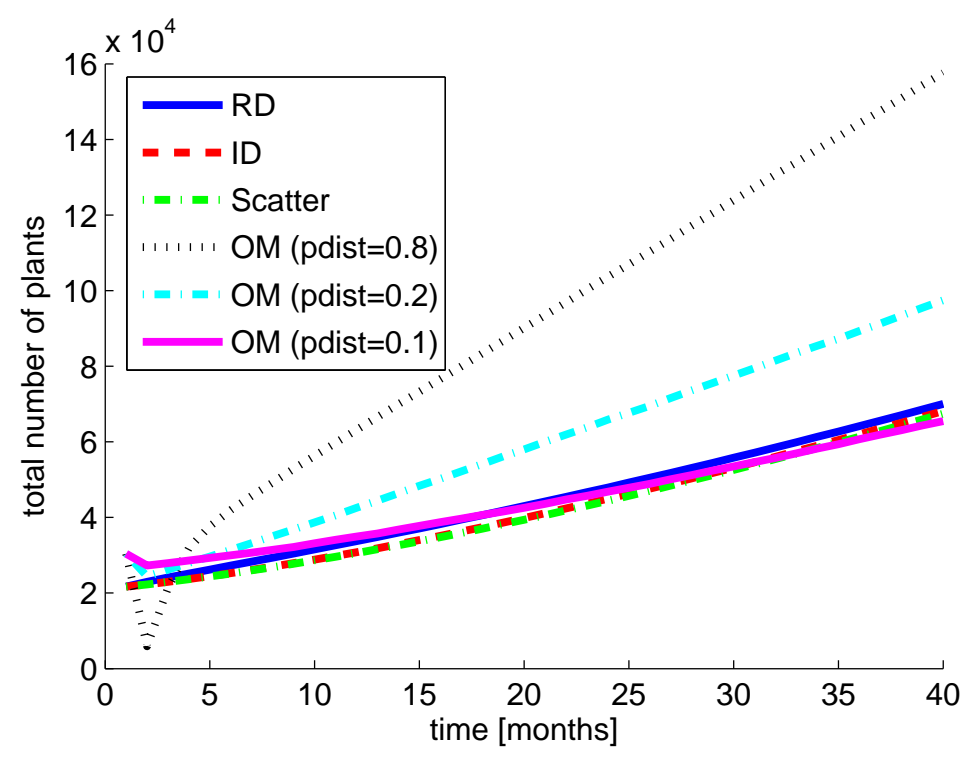

Figure 3: Total number of plants as a function of time: $R D=$ reaction diffusion; ID = integro-difference; $\mathrm{OM}=$ occupation model. 


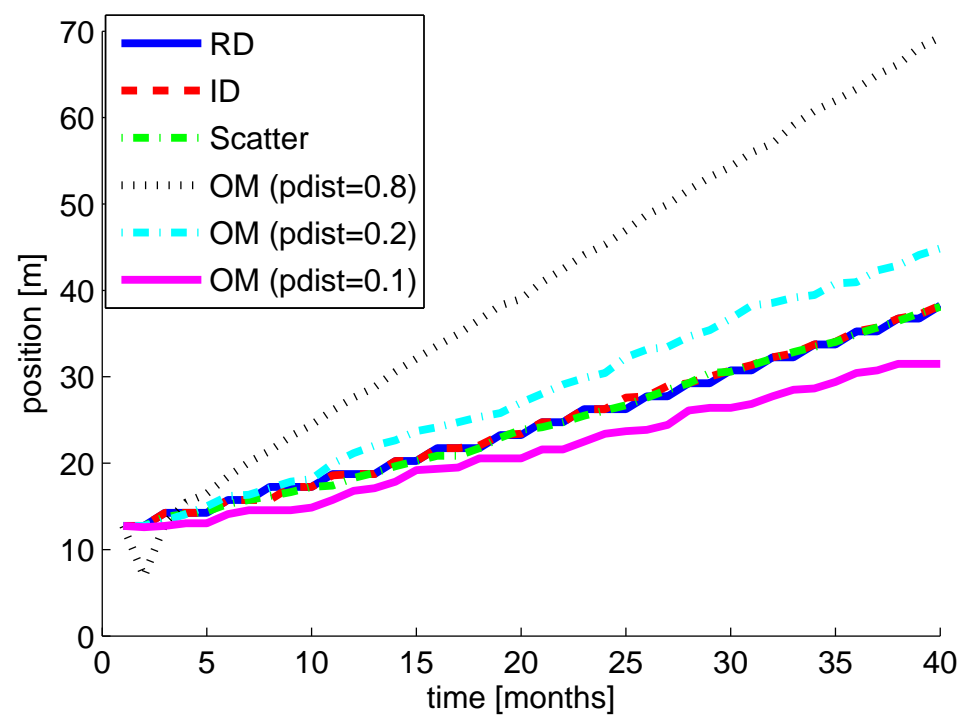

FiguRE 4: Position of the moving weed front for different models.

free boundary at the bottom where plants are leaving the region. The total number of plants is calculated differently for the occupation model since $N=0$ or 1 , where the total number of sites occupied is multiplied by the carrying capacity, $C=20$ branches $/ \mathrm{m}^{2}$, as used in the other models. An interesting result shown in Figure 3 is that as we lower the probability of disturbance $p_{\text {dist }}$, the occupation model better matches the other models, becoming a good match at $p_{\text {dist }}=0.1$.

The standard deviations for Figure 4 were 0.80 metres for the scatter model, for the occupation model with $p_{\text {dist }}=0.8$ it was 0.71 metres, for $p_{\text {dist }}=0.2$ it was 2.6 metres, and for $p_{\text {dist }}=0.1$ it was 2.9 metres. Figure 4 shows the position of the travelling weed front with the reaction diffusion, integro-difference and scatter models well matched. The occupation model with $p_{\text {dist }}=0.8$ progresses one grid at most time steps, resulting in the predominately linear relationship shown. However, as we further decrease $p_{\text {dist }}$ 


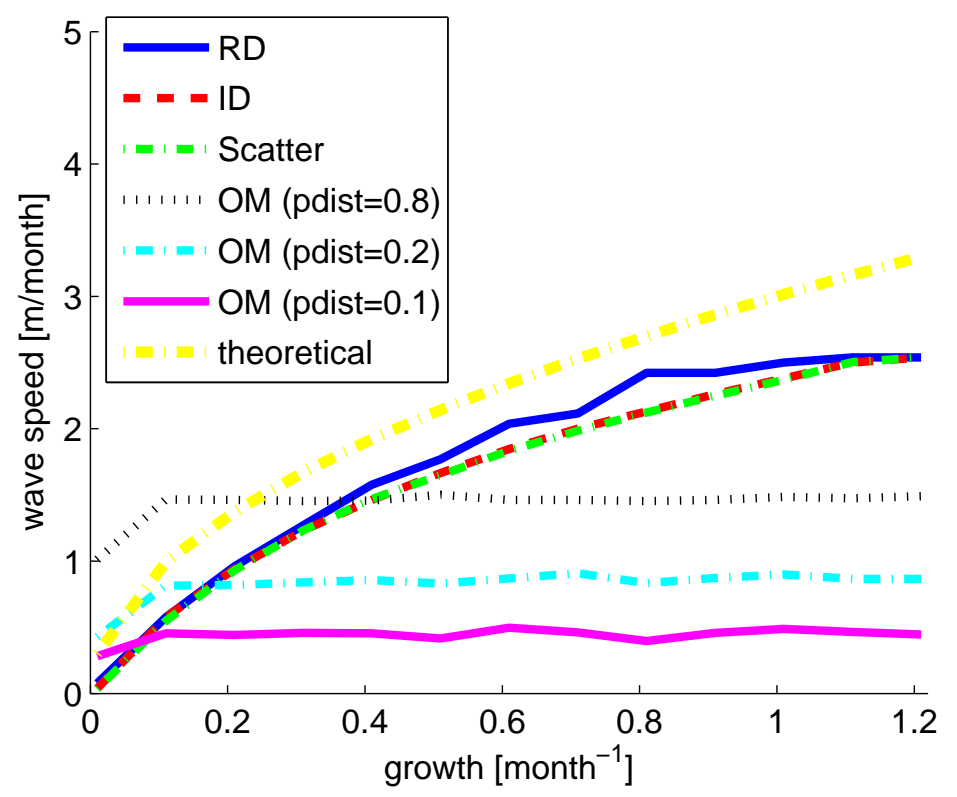

FiguRE 5: Weed front speed as a function of growth for different models.

the element of stochasticity increases, resulting in more fluctuations. Interestingly, where $p_{\text {dist }}=0.1$ was a good match between the occupation and other models for the total plant population, $p_{\text {dist }}=0.2$ is a better match for the travelling weed front.

The standard deviations for Figure 5 were $0.027 \mathrm{~m} / \mathrm{month}$ for the scatter model, for the occupation model with $p_{\text {dist }}=0.8$ it was $0.045 \mathrm{~m} / \mathrm{month}$, for $p_{\text {dist }}=0.2$ it was $0.080 \mathrm{~m} / \mathrm{month}$, and for $p_{\text {dist }}=0.1$ it was $0.096 \mathrm{~m} / \mathrm{month}$. Figure 5 shows the speed of the weed front as a function of the growth term where the reaction diffusion, integro-difference and scatter models are matched. In contrast, the occupation model has very different behaviour with an asymptote evident. This is due to the deficiency in the cellular automata construct as the nearest neighbour rules artificially restrict the dispersal of the weed. The decrease in wave speed with decreasing $p_{\text {dist }}$ is in 
agreement with the results shown in Figure 2. These results do not match the theoretical calculation since the latter requires time frames of the order of 17 years, whereas the time frame for eradication of weeds is in the order of 4 years. Hence the simulations were only run to forty months. We have verified that our numerical implementations reach the theoretical speed, but the results are not shown here.

\section{Conclusion}

We expected, and found, the reaction diffusion, integro-difference and scatter models to be consistent when Gaussian kernels were used, which is a test of the accuracy of our numerical implementations. The parameters for the occupation model could be matched for Figure 3 or Figure 4, but not exactly for both. We also found the speed of the weed front for the occupation model was severely limited by the nearest neighbour distribution kernel and the effect of the probability of disturbance is significant. Further work is required in modelling, measuring and analysing the effect of finite stochastic scatter on 'clumpiness' and hence propagation speed, as well as characterising how the occupation model parameters affect results compared with the other models.

Acknowledgements Roslyn Hickson is supported by a scholarship from the School of Physical, Environmental and Mathematical Sciences, UNSW at ADFA. The assistance of Drs Geoffry Mercer and Harvinder Sidhu is appreciated. 


\section{References}

[1] K. A. Andow, P. M. Kareiva, S. A. Levin and A. Okubo. Spread of invading organisms. Landscape Ecology, 4(2/3), 177-188, 1990. doi:10.1007/BF00132860 C325, C327

[2] B. Baeumer, M. Kovacs, M. M. Meerschaert. Fractional reaction-diffusion equation for species growth and dispersal. To appear in Journal of Mathematical Biology, 2007. http://www. maths. otago.ac.nz/ mcubed/JMBseed.pdf C328

[3] S. I. Barry, R. I. Hickson, K. Stokes. Modelling Lippia spread down flooding river systems. To appear in ANZIAM J. (E), 2007. C325

[4] F. van den Bosch, J. A. J. Metz, O. Diekmann. The velocity of spatial population expansion. Journal of Mathematical Biology, 28, 529-565, 1990. doi:10.1007/BF00164162 C325

[5] H. Caswell. Matrix population models: construction, analysis and interpretation. USA: Sinauer Associates, Inc. 2nd edition, 2001.

[6] R. A. Fisher. The wave of advance of advantageous genes. Annals of Eugenics, 7, 353-369, 1937. http://digital.library.adelaide. edu.au/dspace/bitstream/2440/15125/1/152.pdf C325

[7] A. Hastings. Models of spatial spread: is the theory complete? Ecology, 77(6), 1675-1679, 1996. http://www . jstor .org/view/00129658/di986021/98p0171i/0 C325, C327

[8] S. I. Higgins, D. M. Richardson. A review of models of alien plant spread. Ecological Modelling, 87, 249-265, 1996. doi:0304-3800(95)00022-4 C325, C326, C327, C330 
[9] M. Kot, M. A. Lewis, P. van den Driessche. Dispersal data and the spread of invading organisms. Ecology, 77(7), 2027-2042, 1996. http://www. jstor.org/view/00129658/di986022/98p0217b/0 C325, C326, C329

[10] M. Kot. Elements of mathematical ecology. UK: Cambridge University Press, 2001. C325, C326, C327, C328

[11] M. A. Lewis, M. G. Neubert, H. Caswell, J. S. Clark, K. Shea. A guide to calculating discrete-time invasion rates from data. Conceptual ecology and invasion biology, 1, 169-192, 2006. http:

//www.math.ualberta.ca/ mlewis/publications/Cadotte.pdf C325, C326

[12] M. A. Lewis, S. Pacala. Modeling and analysis of stochastic invasion processes. Journal of Mathematical Biology, 41, 387-429, 2000. doi:10.1007/s002850000050 C328, C330

[13] R. Lui. A nonlinear integral operator arising from a model in population genetics. I. monotone initial data. SIAM Journal of Mathematical Analysis, 13(6), 913-937, 1982. doi:10.1137/0513064 C326

[14] M. G. Neubert, H. Caswell. Demography and dispersal: calculation and sensitivity analysis of invasion spread for structured populations. Ecology, 81(6), 1613-1628, 2000.

http://www. jstor.org/view/00129658/ap010022/01a00120/0 C326, C327, C328, C329

[15] M. G. Neubert, I. M. Parker. Projecting rates of spread for invasive species. Risk Analysis, 24(4), 817-831, 2004. doi:10.1111/j.0272-4332.2004.00481.x C325, C326, C327, C328, C329

[16] A. Okubo. Diffusion and ecological problems: mathematical models. New York: Springer-Verlag, 1980. C325, C326, C327 
[17] M. Rees, Q. Paynter. Biological control of Scotch Broom: modelling the determinants of abundance and the potential impact of introduced insect herbivores. Journal of Applied Ecology, 34, 1203-1221, 1997. http://www. jstor.org/view/00218901/di996085/99p0309w/0 C326, C330, C331

[18] J. G. Skellam. Random dispersal in theoretical populations. Biometrika, 8, 196-218, 1951. doi:10.1007/BF02464427 C325

[19] H. F. Weinberger. Asymptotic behavior of a model in population genetics. In: J. M. Chadam (ed) Lecture Notes in Mathematics No. 648: Nonlinear partial differential equations and applications, 47-96. Proceedings Indiana 1967-1977 (1978). C326

[20] H. F. Weinberger. Long-time behaviour of a class of biological models. SIAM journal on Mathematical Analysis, 13(3), 323-352, 1982. doi:10.1137/0513028 C326 


\section{Author addresses}

1. R. I. Hickson, School Physical, Environmental \& Mathematical Sciences, University of New South Wales at ADFA, Canberra, Australia.

mailto:r.hickson@student.adfa.edu.au

2. S. I. Barry, School Physical, Environmental \& Mathematical Sciences, University of New South Wales at ADFA, Canberra, Australia.

3. K. Stokes, CSIRO Division of Entomology, Canberra, ACT, Australia. 